\title{
VARIATION OF HARMONICS EMISSION FROM MODERN LAMPS IN A POWER SUPPLY NETWORK
}

\author{
RÓBERT ISTÓK ${ }^{* 1}$ \\ ${ }^{1}$ Óbuda University of Budapest, Bécsi út 96/B, Budapest, 1034, Hungary
}

\begin{abstract}
The objective of this paper is to prove the relationship between the Total Harmonics Disturbance emission of lamps network and the number of lamps. In the lighting field the LED lighting emission diode is the absolutely winner. The low power consumption and the characteristics of LED made it ideally for most of indoor and outdoor applications. The dimmability of the LEDs which is between $0 \%$ and $100 \%$ made it ideally for intelligent lighting applications. This large dimmability range could be obtained only with incandescent and halogen bulbs, compare to CFL compact fluorescent lamps where the maximum dimmability is around of $60 \%$. One of the prices of this positive characteristics of modern lamps, is the electromagnetic compatibility EMC. It is well known that the modern lamps have a common part, and this is the electronic ballast. And as all electronics circuits, the electronic ballasts are an important source of EMC noise emission. The noise emission of electronic ballast is mainly conducted emission type. Start from construction of electronic ballast which contains an AC/DC converter and a buffer capacitor the emission of half harmonics is presented too. The emission of half harmonics is proportionally with the cost of lamps, because the cost of lamps depends by the methods used for noise emission rejection too. Typically the cheaper lamps have a higher noise emission than the expensive lamps. Known of noise emission lamps separately is not enough for characterization of low noise emission from lighting network. The low frequency noise emission of a lighting network is lower than in case of a single lamp. The variation of temperature determines a variation of low frequency noise emission too.
\end{abstract}

Keywords: EMC, CFL, LED, Total Harmonic Distortion, EMI filter, conducted noise emission, electronic ballast, half-bridge inverter

\section{INTRODUCTION}

In case of nonlinear loads, the drawn current is not a sinusoidal current. The type of load determines the waveform of the current which should be quite complex, but of course this fact depend by the interaction between the components of the system too. The results of it is a low frequency noise emission in EMC field [1]. With the help of Fourier series analysis [2] the current wave form could be divided into a series of simple sinusoids, which start at the power system fundamental frequency and occur at integer multiples of the fundamental frequency.

The Fourier-type sum is represented as follows [3]:

$$
f(t)=\sum_{n=0}^{\infty}\left(A_{n} \cos \left(n \omega t+\varphi_{n}\right)\right)
$$

\footnotetext{
* Corresponding author, email: istok.robert@kvk.uni-obuda.hu

(C) 2019 Alma Mater Publishing House
} 
$f(t)$ is time dependent periodic function, $A_{n}$ is the amplitude, $\varphi_{n}$ is the phase shift of the $\mathrm{n}$-th harmonics, $\mathrm{n}$ is the number of harmonics, $\omega$ is the angular frequency.

The Fourier-type sum with help of mathematic can be expressed as follows:

$$
f(t)=A_{0}+\sum_{n=1}^{\infty} a_{n} \cos (n \omega t)+\sum_{n=1}^{\infty} b_{n} \sin (n \omega t)
$$

For direct transformation is:

$$
F(\omega)=\int_{-\infty}^{\infty} f(t) e^{-j \omega t} d t
$$

Inverse transformation is:

$$
f(t)=\frac{1}{2 \pi} \int_{-\infty}^{\infty} F(\omega) e^{j \omega t} d \omega
$$

$F(\omega)$ is the spectrum.

The current harmonics trough the impedance of the source distorts the voltage from power source. In case when the impedance of the voltage source is low, the voltage harmonics produced by current harmonics will be low level. This is the case when voltage harmonics are small compared to current harmonics. This situation appears in case of modern lighting lamps, where the voltage half harmonics are only some percent from main voltage.

Total Harmonic Distortion is a measurement of the level of harmonic distortion present in power systems. Total harmonic distortion can be related to either current harmonics or voltage harmonics, and it is defined as the ratio of total harmonics to the value at fundamental frequency times 100\% [4].

$$
\begin{gathered}
T H D_{V}=\frac{\sqrt{V_{2}^{2}+V_{3}^{2}+\cdots+V_{n}^{2}}}{V_{1}} \cdot 100 \%=\frac{\sqrt{\sum_{k=2}^{n} V_{k}^{2}}}{V_{1}} \cdot 100 \% \\
T H D_{I}=\frac{\sqrt{I_{2}^{2}+I_{3}^{2}+\cdots+I_{n}^{2}}}{I_{1}} \cdot 100 \%=\frac{\sqrt{\sum_{k=2}^{n} I_{k}^{2}}}{I_{1}} \cdot 100 \%
\end{gathered}
$$

where $V_{n}$ is the RMS voltage of k-th voltage harmonic; $I_{n}$ is the RMS current of the k-th current harmonic; $\mathrm{k}=1$ fundamental frequency.

\section{EXPERIMENTAL SETUP}

\subsection{Effects of harmonics}

\subsubsection{Motors and generators}

In motors and generators, the voltage and current harmonics trough the iron and cooper losses, increase the heating and affects the developed torque and efficiency. The audible noise emission should appear in motor due to harmonic currents. Another effect of harmonic currents is the resultant flux distribution in the air gap. The fifth and seventh harmonics can create mechanical oscillation in a motor or in a turbine-generator combination load system.

\subsubsection{Transformers}

Current harmonics should increase the cooper losses and stray flux. The effect of voltage harmonics appears in iron losses which result is transformer heating increases. The increasing of frequency determines an increasing of loses. 


\subsubsection{Power cables}

The cables could be affected by voltage stress and corona if cables take part in a resonance system, which can produce dielectric failure.

\subsubsection{Capacitors}

The capacitor bank acts as a sink for higher harmonic currents and the reactance decrease with frequency. This effect increases the heating and dielectric stresses.

\subsubsection{Electronic equipment}

Electronic devices, which are sensitive to the zero-crossing point of the voltage waveform, can be affected by harmonic distortion. Harmonics can shift the voltage zero crossing or the point at which one phase-to-phase voltage becomes greater than another phase-to-phase voltage.

Other types of electronic devices can be affected by transmission of ac supply harmonics through the equipment power supply or by magnetic coupling of harmonics, which can produce malfunctions of device. The harmonic voltage distortion factor should be no more than $5 \%$ and the largest single harmonic no more than 3\% [5].

\subsection{Measurement system}

The standard which describe the harmonic measurements method and the limits for harmonics emission in case of lighting equipment is IEC 61000-3-2. The diagram block of measurement system is presented in Figure 1.

An important requirement for power supply of the measurement system is to be a 'clean supply' with a stable voltage, frequency and poor harmonic emission. This requirement assure that the measured results are come from lighting equipment and not from power supply.

The rated voltage of the device under test determine the voltage of power supply, these two voltages have to be same. In the case of $220 \mathrm{~V}-240 \mathrm{~V}$ voltage range, the test voltage has to be $230 \mathrm{~V}$. The variation of test voltage has to be within $\pm 2.0 \%$ and the frequency within $\pm 0.5 \%$ of the nominal value.

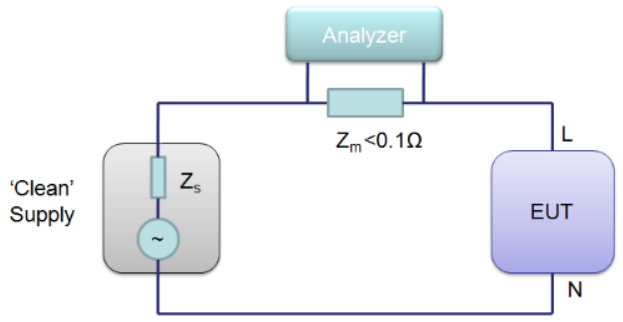

Fig. 1. Diagram of harmonic emission measurement system.

The harmonic emissions must not exceed the next values:

- $0.9 \%$ for harmonic of order 3 ;

$-0.4 \%$ for harmonic of order 5 ;

$-0.3 \%$ for harmonic of order 7 ;

$-0.2 \%$ for harmonic of order 9 ;

- $0.2 \%$ for even harmonics of order from 2 to 10 ;

- $0.1 \%$ for harmonics of order from 11 to 40 .

The peak value of the test voltage shall be within 1.40 and 1.42 times its RMS value and shall be reached within $87^{\circ}$ to $93^{\circ}$ after the zero crossing [6]. The EUT (equipment under test) in this case is a lighting lamp.

\subsection{Total Harmonics Distortion emission}

Typically, the standards require measurements of only one lamp, but in real life more than one lamp are using in a network. This fact determines the need for measurements of lighting networks. The reason why the standards require measurement of only one lamp is that the repeatability of measurements must be assured. The combination of different lamps should produce different measurement results. To understand the mechanism of harmonics emission let is start from the typically circuit diagram of an electronic ballast which is presented in Figure 2. The input voltage is $220-240 \mathrm{VAC} 50 / 60 \mathrm{~Hz}$, the first circuit part is the EMI filter the role of it is to block the noise emission, the next part is a diode rectifier with smoothing capacitor, a half-bridge inverter for DC 
to AC conversion, and the resonant circuit to ignite and run the lamp. In this case the main source of low frequency harmonics is the rectifier stage [7].

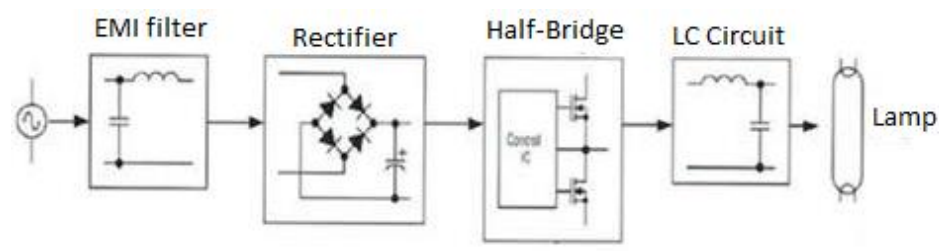

Fig. 2. Diagram circuit of electronic ballast.

In Figure 3 the voltage and current wave form of a $11 \mathrm{~W}$ compact fluorescent lamp, in Figure 4 the measurement results of a $23 \mathrm{~W}$ induction lamp are presented. The wave form of current is not sinusoidal, which means that the harmonic current emission has a high level. The amount of harmonic emission is higher when the increase of current waveform starts later than voltage wave form. One of standard's requirement is that current has to start flowing before $60^{\circ}$. The level of harmonic emission in case of induction lamp is higher than emission of compact fluorescent lamp.

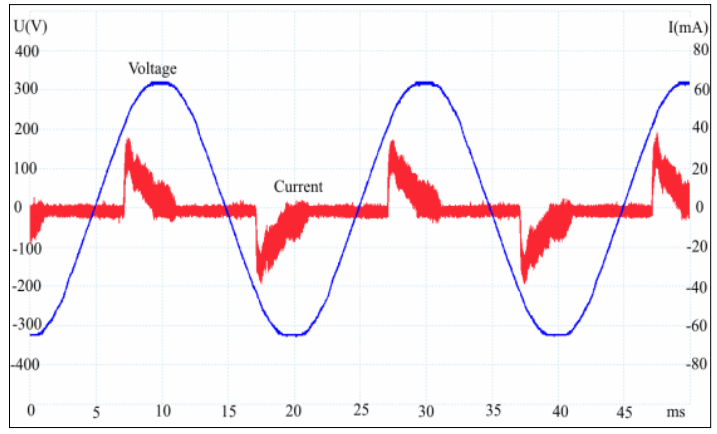

Fig. 3. Current and voltage wave form of a compact fluorescent lamp.

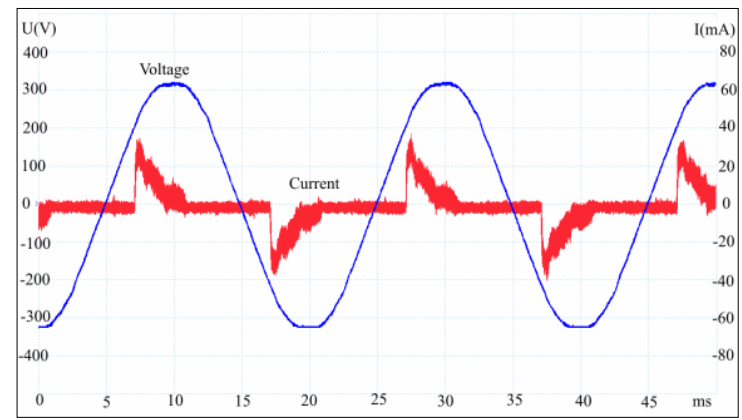

Fig. 4. Current and voltage wave form of a $23 \mathrm{~W}$ induction lamp.

Power Factor PF and Total Harmonic Distortion provide a quantitative measure of the power quality in an electrical system. The amount of useful power being consumed by an electrical system is predominantly decided by the Power Factor of the system [8].

The Power Factor Correction in case of cheap LEDs and compact fluorescent lamps is done with inductors which are used on DC side or AC side, Figure 5 and Figure 6.

$C_{i}$ - capacitor could improve the power factor. 
In case of expensive LED lamps, the harmonic emission in lower than in case of CFL lamps [8, 9]. The reason is that the power factor correction is done with active power factor correction circuits. This solution should be applied for CFL lamps too, but it produces an increasing of price. For a comparison in Figure 7 is presented the wave forms of current and voltage of a 9W LED and a 7W LED lamp. The current wave form of 7W LED is close to sinusoidal shape what is mean the harmonics emissions are on a low level. The current wave form of $9 \mathrm{~W}$ LED lamp has spike form, what is mean this lamp has a high harmonic emission. There is a correlation between the price of lamps and harmonic emission. The lamps whit a higher price typically has a lower harmonic emission.

For higher quality and more expensive LED lamps a better power factor is achieved with active power factor correction circuits [10]. In Figure 8 is presented the measurement results for a 15W LED with good power factor correction and a $15 \mathrm{~W}$ LED with poor factor correction. The voltage total harmonic distortion is very low in case of modern lightings. The LED with low power factor correction has a spike current wave form which is similar with current wave form of compact fluorescent lamps. The reason is that in case of cheaper LED lamps the electronic ballast is very similar with the electronic ballast used for compact fluorescent lamps except the module used for igniting of fluorescent tube. In Figure 9 is presented the measurement results of a $23 \mathrm{~W}$ and a $22 \mathrm{~W}$ compact fluorescent lamps. There is a similarity between the current wave forms, but the in case of $22 \mathrm{~W}$ lamp the current start to increase later than in case of $23 \mathrm{~W}$ lamp, that is mean the harmonic emission of $22 \mathrm{~W}$ lamp is higher. The second observation is that the current of $22 \mathrm{~W}$ lamp is much lower, that is mean the wattage of the lamp is lower than the wattage specified on the lamp.

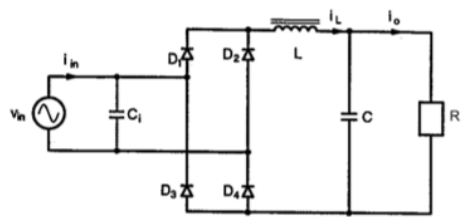

Fig. 5. Full-wave rectifier with LC filter dc-side inductor.

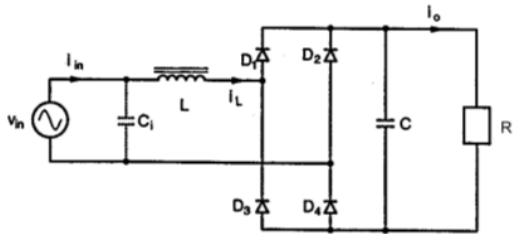

Fig. 6. Full-wave rectifier with LC filter ac-side inductor.

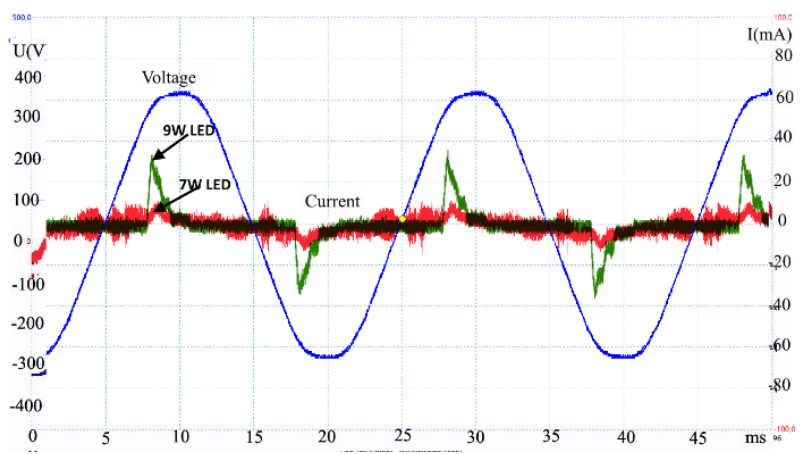

Fig. 7. Current and voltage wave form of a 9W LED and a 7W LED. 


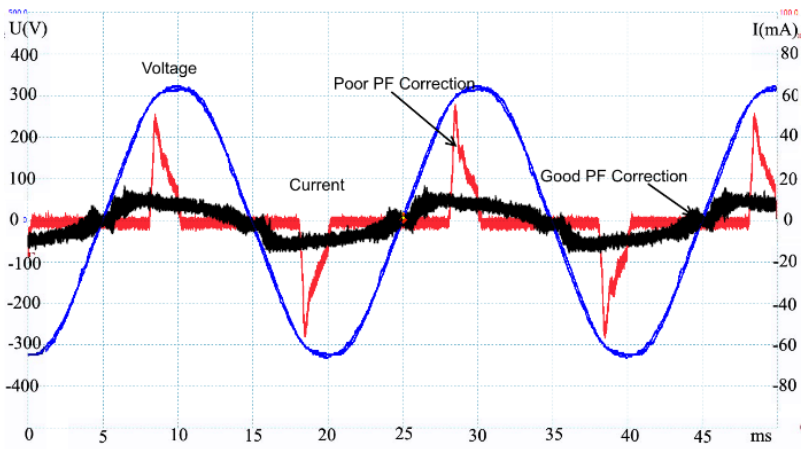

Fig. 8. Current and voltage wave form of a 15W LED with good PF correction and a 15W LED with poor Power Factor Correction.

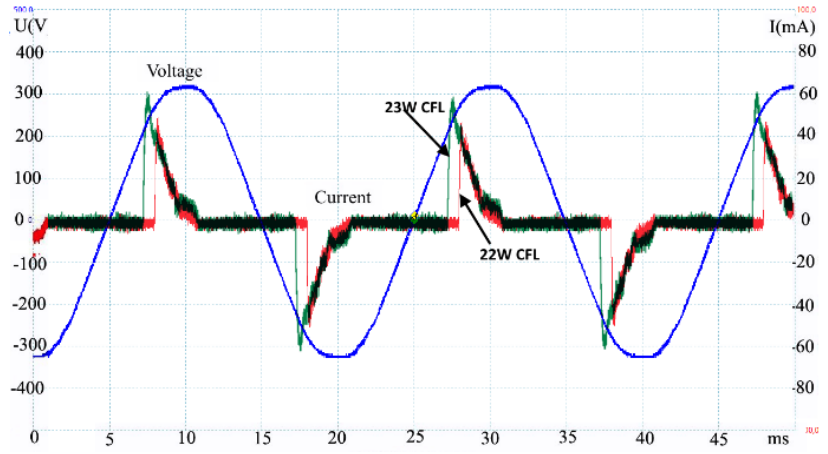

Fig. 9. Current and voltage wave form of a $23 \mathrm{~W}$ and $22 \mathrm{~W}$ compact fluorescent lamps.

\section{MEASUREMENT RESULTS OF LIGHTING NETWORKS}

As mentioned before, the standard measurement required only one lamp to be measured, but in an office for example there are more than one lamp. In Figure 10 can be seen the variation of a low frequency noise emission function of lamps number variation.

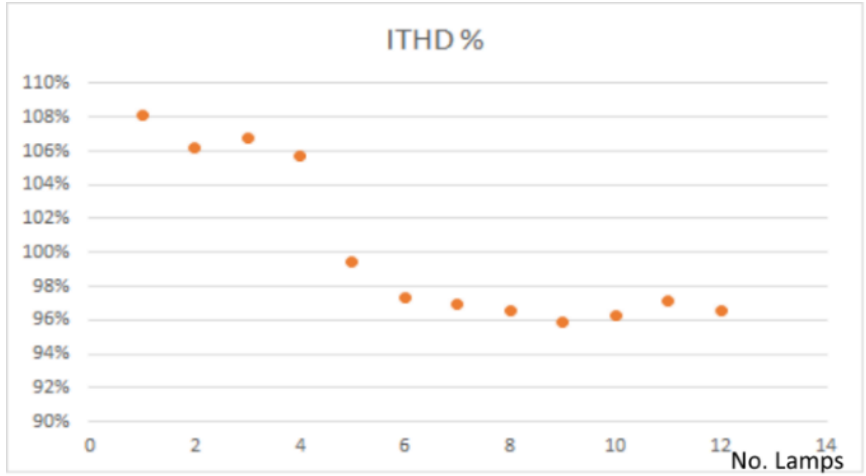

Fig. 10. Total Harmonic Distortion emission for different number of compact fluorescent lamps.

The measurement results show that, increasing of number of compact fluorescent lamps, determine the Total Harmonic Distortion noise emission decreasing from $108 \%$ to $96 \%$. The Total Harmonic Distortion noise emission became quite stable around of 8 lamps. Increasing of lamps number over 8 does not produce a significantly modification of noise emission. The result of this measurement show that the increasing of lamps number does not determine an increasing of noise emission on the contrary it will be lower than in case of one lamp. 
All the standards which refer to the lighting lamps state that before starts the measurements the lamps have to function for minimum 15 minutes. The reason for this time period is that the during this time the all parameters of lamps are stabilized. The temperature during the measurements required by standard have to be quite constant. The stabilization of parameters and the constant temperature assure the repeatability of measurements fact which is very important.

In Figure 11 the Current Total Harmonic Distortion emission was measured at different ambient temperature. The measurements were done with cold lamp, that is mean the lamps were switched on and after 30 seconds the measurement was done. Variation of temperature determine a variation of noise emission too. During the measurements the lamps network contained 6 LED lamps.

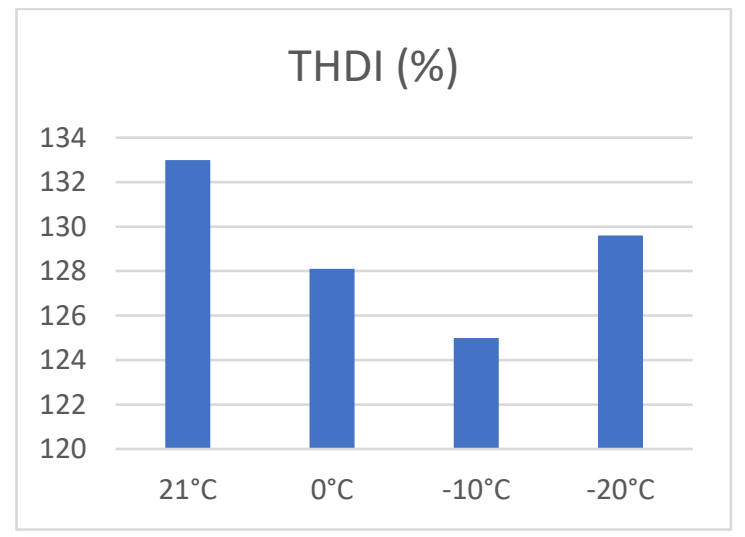

Fig. 11. Variation of Current Total Harmonic Distortion emission with variation of temperature.

In Figure 12 is presented the Current Total Harmonic Distortion emission variation function of working temperature variation of lamp. The noise emission of lamps depends by working temperature of lamps. After switching on the lamps, the noise emission is changing function of working temperature change.

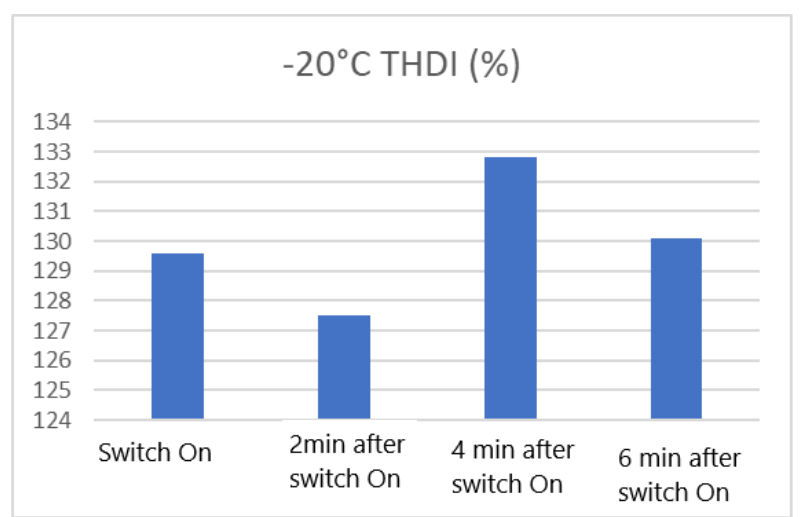

Fig. 12. Variation of Current Total Harmonic Distortion emission with variation of lamps working temperature.

\section{CONCLUSIONS}

Some of modern lamps type have an important harmonic emission. Harmonic emission of some cheaper LED lamps could be higher than emission of compact fluorescent lamps. The differences between Total Harmonic Distortion emissions in case of different compact fluorescent lamps are significantly, but the differences are not so high than in case of LED lamps. Increasing of lamps number determine a decreasing of Total Harmonic Distortion emission compare to Total Harmonic Distortion emission from a single lamp. In case of network which contains CFL lamps the noise reduction is stronger than in case of network which contains LED lamps. The Total Harmonic Distortion decreasing is valid only till 8 lamps. If the number of lamps is increasing over 8 the Total Harmonic Distortion emission remain approximately constant. This relation between number of lamps and the Total Harmonic Distortion emission is valid for CFL and LED lamps too. That is mean the fluorescent tube and the LED chips do not influence the emissions. Another results this study is that the variation of temperature has an influence on the Total Harmonic Distortion emission of lamps too. 


\section{REFERENCES}

[1] Azcarate, J.J., Gutierrez, A., Lazkano, P., Saiz, K., Redondo, L.A., Experimental study of the response of efficient lighting technologies to complex voltage fluctuations, International Journal of Electrical Power and Energy Systems, vol. 63, 2014, p. 499- 506.

[2] Abagiu, S., Lepadat, I., Helerea, E., Mihai, C., Assesment of voltage quality indicators in unbalanced and harmonic distorted regime, Pollack Periodica, vol. 11, no. 1, 2016, p. 67-79.

[3] Bystrik, D., Contribution to the electromagnetic compatibility of prototype LED street light, The 8th International Scientific Symposium Elektroenergetika 2015, Stará Lesná, Slovak Republic, 16-18. 09. 2015, p. 307-310.

[4] Domagk, M., Zyabkina, O., Meyer, J., Schegner, P. Trend identification in power quality measurements, 2015 Australasian Universities Power Engineering Conference, Wollongong, Australia 27-30 September 2015, p. $1-6$.

[5] IEEE Std 519, IEEE recommended practices and requirements for harmonic control in electrical power systems, 2014.

[6] IEC 61000-3-2, Electromagnetic compatibility (EMC) - Part 3-2, Limits for harmonic current emissions (equipment input current $\leq 16$ A per phase), 2014.

[7] Koponen, P., Hansen, H., Bollen, M., Inter-harmonics and light flicker, International Conference on Electricity Distribution, Lyon, France, 15-18 June 2015, paper 1100.

[8] Gil-de-Castro, A., Rönnberg, S., Bollen, M.H.J., Moreno-Muñoz, A., Pallres-Lopez, V., Harmonics from a domestic customer with different lamp technologies, 2012 IEEE 15th International Conference on Harmonics and Quality of Power, Hong Kong, 17-20 June 2012, p. 585 - 590.

[9] Rönnberg, S.K., Wahlberg, M., Bollen, M.H.J., Harmonic emission befor and after changing to LED lamps - Field measurements for an urban area", 2012 IEEE 15th International Conference on Harmonics and Quality of Power, Hong Kong, 17-20 June 2012, p. 552-557.

[10] Power Factor Correction (PFC) basics, Fairchild semiconductor, Application note 42047, 2004. 